\title{
CAE-based application for identification and verification of hyperelastic parameters
}

\author{
Yevgen Gorash;, Tugrul Comlekci and Robert Hamilton \\ Department of Mechanical \& Aerospace Engineering, University of Strathclyde, James Weir Building, 75 \\ Montrose Street, Glasgow G1 1XJ, UK
}

\begin{abstract}
The main objective of this study is to develop a CAE-based application with a convenient GUI for identification and verification of material parameters for hyperelastic models available in the current release of the FE-code ANSYS Mechanical APDL. This Windows-application implements a 2-step procedure: 1) fitting of experimental stress-strain curves provided by user; 2) verification of obtained material parameters by the solution of a modified benchmark problem. The application, which was developed using Visual Basic .NET language, implements a two-way interaction with ANSYS as a single loop using the APDL-script as an input and text, graphical and video files as an output. With this application, 9 isotropic incompressible hyperelastic material models are compared by fitting them to the conventional Treloar's experimental dataset (1944) for a vulcanised rubber. The ranking of hyperelastic models is constructed according to the models efficiency, which is estimated using a fitting quality criterion. The models ranking is done based upon the complexity of their mathematical formulation and ability of accurately reproducing the test data. Recent hyperelastic models (Extended Tube and Response Function) are found more efficient compared to conventional ones. The verification is done by the comparison of an analytical solution to a FEA result for the benchmark problem of rubber cylinder under compression proposed by Lindley (1967). In the application, the classical formulation of the benchmark is improved mathematically to become valid for larger deformations. The wide applicability of the proposed 2-steps approach is confirmed using stress-strains curves for 7 different formulations of natural rubber and 7 different grades of synthetic rubbers.
\end{abstract}

\section{Keywords}

Benchmark, elastomer, Finite Element Analysis, hyperelasticity, ranking, software

\section{Introduction}

This study addresses nine isotropic incompressible hyperelastic models for rubber-like materials, which are available in the current release of FE-code ANSYS $\stackrel{1}{1}$. ANSYS Mechanical APDL v. 15.0 has been chosen for the implementation of the WARC research project $\mathrm{C}_{2}^{2} \underset{2}{ }$, since it has been a leading CAE product for FE-analysis for over 40 years. Moreover, it is capable of all the essential FE-simulation features required for the analysis of elastomeric components and comprises recently developed hyperelastic models. Totally, ANSYS includes the following models 1 :
- Nearly-incompressible isotropic models: NeoHookean ${ }^{3,4}$, Mooney-Rivlin 5,6, Polynomial Form? ${ }^{7}$, Ogden Potential $\stackrel{-8}{ }$, Arruda-Boyce $\stackrel{9}{=}$, Gent $\stackrel{10}{ }$, Yeoh $\stackrel{11}{ }$, and Extended Tube 12 .

- Compressible isotropic models: Blatz-Ko $\underline{13}$ and Ogden Compressible Foam 14 options are applicable to compressible foam or foam-type materials.

- Nearly-incompressible isotropic response function hyperelastic model. The "response function" model in ANSYS is equivalent to the Marlow model 15 implemented in ABAQUS and Sussman-Bathe model 16 implemented in ADINA. This model is an exception

\footnotetext{
${ }^{*}$ Corresponding author. Tel.: +44 790 9780901; E-mail: yevgen.gorashestrath.ac.uk. URL: www.strath.ac.uk/mae/
} 
from classification, because it obtains the constitutive response functions directly from experimental data.

- Invariant-based anisotropic strain-energy potential.

It should be noted that anisotropic and compressible isotropic models are out of the scope of this study.

Over the last decade, a significant number of reviews and comparative studies on hyperelastic constitutive models has been published. Availability of these studies is caused by a great choice of hyperelastic material models and recommendations $\underline{17-19}$ for their selection and application in FEA. To describe the elastic behaviour of rubber-like materials, numerous specific forms of strain energy functions have been proposed in the literature so far. They are permanently evolving and improving in mathematical formulations, because of a great demand for a reliable constitutive model to be used in FE-simulations for a variety of applications. Generally, these studies address the ability of hyperelastic models to capture the complex behaviours of rubber-like materials including the material model stability aspect and quality of experimental data fitting. Seibert and Schöche 20 compared six different models using their own experimental data obtained with uniaxial and biaxial tension tests on the $17 \mathrm{wt} \%$ carbon black-filled HNBR rubber. Boyce and Arruda 21 compared five models using the classical data set by Treloar ${ }^{22}$ for uniaxial and biaxial tension and pure shear tests on $8 \% \mathrm{~S}$ vulcanised rubber. Xia et al. ${ }^{23} \mathrm{com}$ pared three compressible hyperelastic models using their own experimental data obtained with uniaxial tension tests on five variants of rubber compounds. Chagnon et al. ${ }^{24}$ compared three recent models using Treloar's ${ }^{22}$ set of experimental data. Attard \& Hunt ${ }^{25}$ considered experimental data of different authors for five different deformation modes to demonstrate the efficiency of their proposed model. Marckmann \& Verron ${ }^{26}$ published a thorough comparison of twenty hyperelastic models using two classical sets of experimental data - Treloar's 22 and biaxial extension of the sheet specimens made of isoprene rubber vulcanizate by Kawabata et al. ${ }^{27}$. Moreover, a ranking of these twenty models with respect to their ability to fit test data is established by Marckmann \& Verron ${ }^{26}$, highlighting new efficient constitutive equations that could advantageously replace wellknown models. The corresponding material parameters for both sets of experimental data 22,27 are identified using own fitting procedure and reported in Ref. $\underline{\underline{26}}$ Ruíz \& González $\underline{28}$ present a review of the application of hyperelastic models to the analysis of fabrics using FEA. For this purpose seven models available in ANSYS were compared using own experimental data obtained with uniaxial and biaxial tension and simple shear tests on a fabric. In result, a comparison and ranking of models were implemented through the 3D benchmark problem of a rigid body in contact with a hyperelastic fabric. Vahapoğlu \& Karadeniz $\underline{29}$ provided a comprehensive bibliography $(1930-2003)$ containing a list of references on the strain energy functions for rubberlike materials on isothermal condition. The classification of models 29 includes eighty seven material models, and it is based on either specific strain energy function formulations or the discussions on such suggested forms using the phenomenological approach. Another bibliographic review on constitutive models used in FEA packages for analysis of rubber components was proposed by Ali et al. ${ }^{30}$ Dimitrov 31 discussed three classes of hyperelastic models (phenomenological, response-type and micromechanical), which are available in ANSYS 13. The ranking 31 of models is also proposed according to their capability of accurately reproducing the multiaxial loading states observed in reality. $\mathrm{Li}$ et al. ${ }^{32}$ compared classical Mooney-Rivlin $\underline{5,6}$ and Ogden $\underline{\underline{8}}$ models using their own experimental data obtained with uniaxial, biaxial and planar tension tests on natural rubber specimens filled with $46 \mathrm{phr}$ carbon black. One of the most recent and comprehensive comparative studies of hyperelastic models is presented by Steinmann and his co-workers 33,34 . They provided both accurate stress tensors and tangent operators for a group of totally twenty five phenomenological and micromechanical models at large deformations (14 conventional models in Ref. $\frac{33}{3}$ and 11 more recent models in Ref. .34 ). For comparison of all selected models in reproducing the well-known Treloar's experimental data ${ }^{22}$, the analytical expressions for the three homogeneous deformation modes (uniaxial tension, biaxial tension and pure shear) have been derived and the performances of the models are analysed in Refs ${ }^{33,34}$. Finally, Beda ${ }^{35}$ developed a mathematical approach for the best way to structure incompressible hyperelastic models, and applied it to the estimation of convectional phenomenological models using Treloar's 22 dataset. 
Table 1. Comparison of estimated parametric errors for all incompressible isotropic hyperelastic material models supported by ANSYS

\begin{tabular}{|c|c|c|c|c|c|c|c|c|c|c|c|c|c|c|c|c|}
\hline \multirow{2}{*}{ no. } & \multirow{2}{*}{ material model } & \multicolumn{15}{|c|}{ number of parameters } \\
\hline & & 0 & 1 & 2 & 3 & 4 & 5 & 6 & 7 & 8 & 9 & 10 & 11 & 12 & 13 & 14 \\
\hline 1 & Mooney-Rivlin & & & 5.192 & 7.677 & & 5.968 & & & & 2.544 & & & & & \\
\hline 2 & Ogden & & & 5.189 & & 3.643 & & 4.381 & & 5.792 & & 1.366 & & 1.964 & & 1.548 \\
\hline 3 & Polynomial & & & 5.192 & & & 5.968 & & & & 2.544 & & & & & 1.166 \\
\hline 4 & Yeoh & & 2.848 & 3.595 & 2.922 & 3.779 & 3.583 & 4.232 & 4.922 & 5.624 & & & & & & \\
\hline 5 & Extended tube & & & & & 0.686 & & & & & & & & & & \\
\hline 6 & Arruda-Boyce & & & 2.499 & & & & & & & & & & & & \\
\hline 7 & Gent & & & 2.161 & & & & & & & & & & & & \\
\hline 8 & Neo-Hookean & & 2.848 & & & & & & & & & & & & & \\
\hline 9 & Response function & 0.184 & & & & & & & & & & & & & & \\
\hline
\end{tabular}

Assessment of hyperelastic models efficiency

\section{Curve fitting tools}

The ANSYS curve fitting tool ${ }^{36}$ is an application embedded into ANSYS for estimating material constants by inputting user's experimental data. Quality of the fitting is assessed by comparing visually the curves obtained with hyperelastic material models to experimental data. User's stress-strain curves can be converted to any of the supported hyperelastic models mentioned above. The curve fitting can be performed either interactively (GUI) or via batch commands by doing the 7 -steps procedure ${ }^{36}$. In this study, ANSYS curve fitting tool is operated in batch mode by the external application using APDL commands.

Alternative curve fitting tools for hyperelastic and other non-linear material models are available as stand-alone applications and add-ins for other CAD/CAE products:

1. MCalibration 37 - a software, which enables semiautomatic extraction of pertinent material parameters from test data for a number of advanced non-linear material models.

2. Hyperfit $\frac{38}{-}$ - a curve fitting utility for automatic parameter identification for a large number of hyperelastic constitutive models.

3. Curve fitting tools incorporated into FE-codes of alternative CAE-systems (e.g. ABAQUS, COMSOL and MSC Marc) and FE-addins of CAD-systems (e.g. Creo Simulate and SolidWorks Simulation).

These tools are different in their functionality, mathematical methods for fitting, number and types of supported material models. All applications support conventional hyperelastic material models like Mooney-Rivlin $\stackrel{5,6}{ }$, Ogden $\stackrel{8}{-}$, ArrudaBoyce $\stackrel{9}{ }$, Gent $\stackrel{10}{ }$, Yeoh $\stackrel{11}{ }$, etc. Some of these tools support more recent advanced material models, which require more computational efforts, like Extended Tube model 12 .

\section{Least squares fit analysis}

The curve fitting process is based upon a regression analysis using the computational method called least squares $\operatorname{method}^{39}$. By performing a least squares fit analysis the material constants can be determined from experimental stress-strain data and constitutive equations for the principal true stress $\sigma_{11}$ under uniaxial and biaxial tension and pure shear correspondingly:

$$
\begin{gathered}
\sigma_{11}=2\left(\lambda_{1}^{2}-\lambda_{1}^{-1}\right)\left[\frac{\partial W}{\partial I_{1}}+\lambda_{1}^{-1} \frac{\partial W}{\partial I_{2}}\right], \\
\sigma_{11}=2\left(\lambda_{1}^{2}-\lambda_{1}^{-4}\right)\left[\frac{\partial W}{\partial I_{1}}+\lambda_{1}^{2} \frac{\partial W}{\partial I_{2}}\right], \\
\sigma_{11}=2\left(\lambda_{1}^{2}-\lambda_{1}^{-2}\right)\left[\frac{\partial W}{\partial I_{1}}+\frac{\partial W}{\partial I_{2}}\right],
\end{gathered}
$$

where $\lambda_{1}-1$ st principal stretch ratio, $W$ - strain energy density function defined by material model, $I_{1}$ and $I_{2}-1$ st and 2nd principal strain invariants correspondingly. Equations (1)-(3) are fitted simultaneously to the available experimental curves. Briefly, the least squares fit minimises the sum of squared error (SSE) between experimental and Cauchy predicted stress values. The sum of the squared error or error norm is defined by:

$$
E r r=\sum_{i=1}^{n} w_{i}\left[\sigma_{i}^{\mathrm{exp}}-\sigma_{i}^{\mathrm{eng}}\left(c_{j}\right)\right]^{2}
$$

where $E r r-S S E$ or least squares residual error; $\sigma_{i}^{\text {exp }}$-experimental stress values; $\sigma_{i}^{\text {eng }}$ - engineering stress values as 
functions of hyperelastic material constants; $n$ - number of experimental data points; and $w_{i}$ - weights associated with different data points, if a non-normalised or weighted error norm is used. For example, if the error in the $i$ th observation is approximately $e_{i}$, then the weight is defined as $w_{i}=1 / e_{i}$. If a normalised (non-weighted) error norm is used for the fit analysis then $w_{i}=1$.

Equation (4) is minimised by setting the variation of the squared error to $\delta E r r^{2}=0$. This yields a set of simultaneous equations which are used to solve for the hyperelastic constants:

$$
\frac{\partial E r r^{2}}{\partial c_{1}}=0, \quad \frac{\partial E r r^{2}}{\partial c_{2}}=0, \quad \ldots \quad \text { etc. }
$$

For the pure shear case, the hyperelastic constants cannot be uniquely determined from Eq. (3). In this case, the shear data must by supplemented by either or both of the other two types of test data to determine the constants using Eqs (1) and (2).

\section{Fitting quality criteria}

In order to choose an optimal or the most efficient hyperelastic model from the variety of the available models based upon the fitting results, some choice criteria are required. These criteria were proposed by Chagnon et al. ${ }^{24}$ as follows:

1. The model should be able to accurately reproduce the whole "S"-shaped form of experimental stress-strain curves at large deformation;

2. The change of deformation modes should not be problematic, i.e. if the model behaves satisfactorily in uniaxial tension, it should also be quite accurate in simple shear or in biaxial extension;

3. The number of relevant material parameters must be as small as possible, in order to reduce the number of experimental tests needed for their identification;

4. The mathematical formulation has to be quite simple to render possible the numerical implementation of the material model.

This list can be extended by recommendations from ANSYS documentation ${ }^{36}$ as follows:

1. Error norm (or SSE), defined by Eq. (4) using least squares fit analysis, should have a minimum value for the model when compared to other less efficient models;
2. A hyperelastic model based on a high-order polynomial for a strain-energy function should pass a stability check.

This requirement ${ }^{36}$ means that a nonlinear material model is stable if the secondary work required for an arbitrary change in the deformation is always positive: $d \sigma_{i j} d \varepsilon_{i j}>0$, where $d \sigma_{i j}$ - change in the Cauchy stress tensor corresponding to a change in the logarithmic strain $d \varepsilon_{i j}$.

Since the simplicity of the material model is as much important as a goodness of fitting provided by it, a new fitting quality criterion is proposed. This criterion is a parametric error $E r r_{\mathrm{p}}$ - product of the error norm Err using Eq. (4) and number of non-zero material parameters in the hyperelastic model $N_{\mathrm{p}}$ :

$$
E r r_{\mathrm{p}}=E r r \cdot N_{\mathrm{p}},
$$

where both $E r r$ and $N_{\mathrm{p}}$ need to be minimised to provide an optimal quality material model. Minimum $N_{\mathrm{p}}$ indicates the simplest material model, while minimum Err indicates the most accurate fitting.

\section{Hyperelastic models ranking}

ANSYS documentation 36 provides the following recommendation for the selection of optimal hyperelastic model according to the strain range $\Delta \varepsilon$ of their applicability:

- Neo-Hookean model is valid at $\varepsilon<30 \%$ (defined by 1 parameter),

- Mooney-Rivlin model is valid at $\varepsilon<100 \%$ (for 2 and 3 parameters) and at $\varepsilon<200 \%$ (for 5 and 9 parameters),

- Polynomial Form model is valid at $\varepsilon<300 \%$ (for 3 rd order),

- Arruda-Boyce and Gent models are valid at $\varepsilon<300 \%$ (both contain 2 parameters),

- Yeoh model is valid at $\varepsilon<300 \%$ (for 3rd order),

- Ogden model is valid at $\varepsilon<700 \%$ (for 3rd order).

This recommendation includes only conventional models and defines the Neo-Hookean model ${ }^{3.4}$ as an optimal choice for a narrow strain range $\Delta \varepsilon$, Arruda-Boyce ${ }^{9}$ and Gent ${ }^{10}$ models - for a moderate $\Delta \varepsilon$, Ogden model ${ }^{\underline{8}}$ - for a wide $\Delta \varepsilon$.

Analogically to comparative studies by Steinmann et al. 33.34, Dimitrov $\stackrel{31}{ }$, Ruíz \& González $\stackrel{28}{ }$, Marckmann \& Verron ${ }^{26}$, an assessment of the fitting quality and efficiency for hyperelastic models was done. The given comparative study is based on Treloar's 22 set of experimental data including uniaxial, biaxial and planar curves. This study of natural 


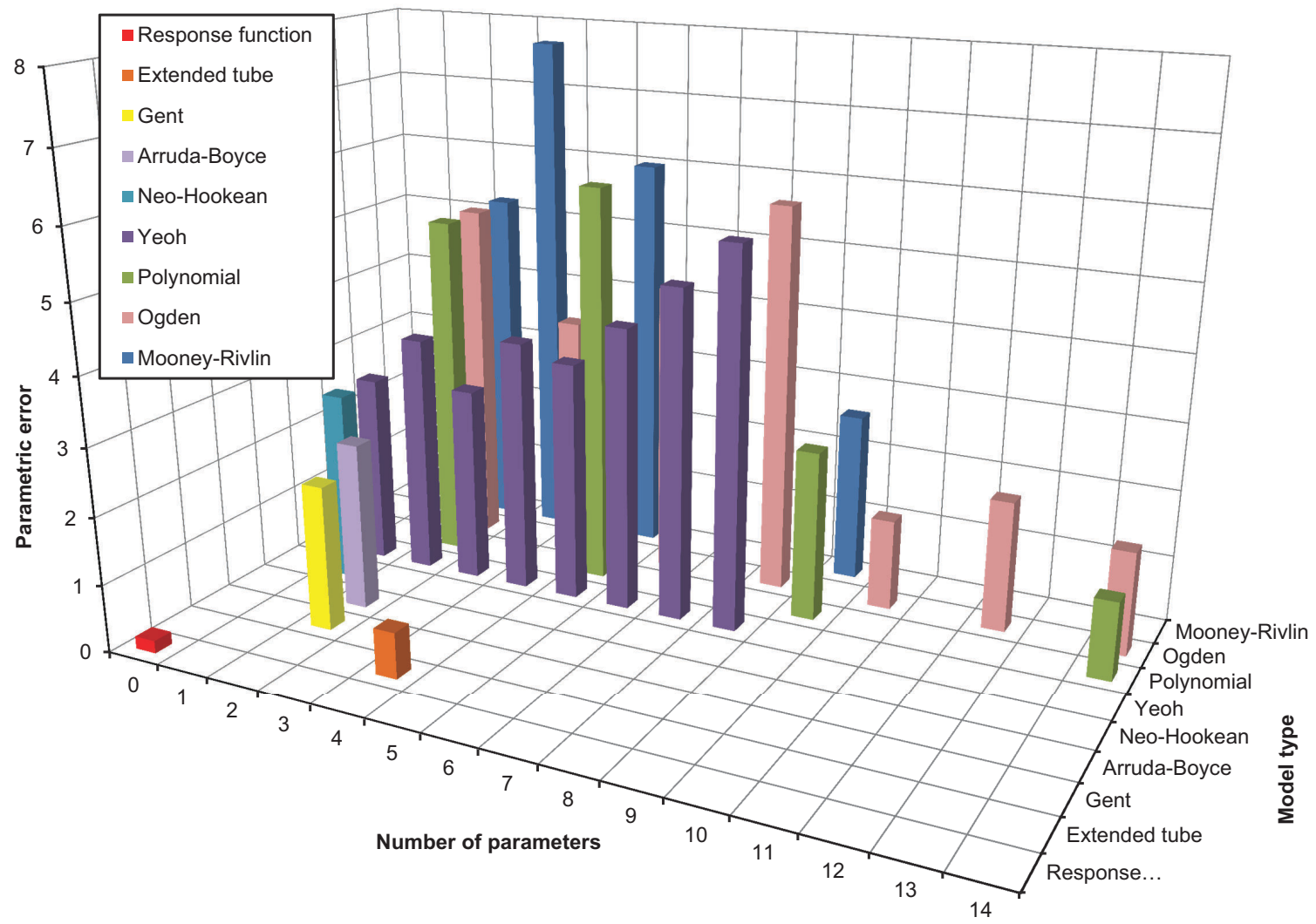

Fig. 1. Comparison of estimated parametric errors for all incompressible isotropic hyperelastic material models supported by ANSYS

vulcanised $8 \% \mathrm{~S}$ rubber is one of the earliest comprehensive experimental studies of elastomers under various types of deformation. This set of data has been used later in many theoretical studies for the formulation, validation and calibration of several hyperelastic models, e.g. Ogden $\stackrel{8}{-}$, ArrudaBoyce $\stackrel{9}{ }$, Extended Tube $\stackrel{12}{ }$, etc. It also has been used as the basis for comparison of material models in many reviews, such as Boyce and Arruda ${ }^{21}$, Attard \& Hunt $^{25}$, Marckmann \& Verron $\stackrel{26}{ }$, Steinmann et al. $\stackrel{33.34}{ }$, Li et al. .32

In contrast to $\stackrel{26,33,34}{ }$, the number of models participating in this assessment was limited to only nine isotropic incompressible models supported by ANSYS for non-linear FEA. Compared to the studies ${ }^{28,31}$ dealing with ANSYS models only, current work employs a strict mathematical criterion (6) for the model assessment and provides a corresponding hyperelastic model ranking presented in Table 1 and illustrated on chart in Fig. 1. Table 1 lists the values of $E r r_{\mathrm{p}}$ for all formulations of the models with different $N_{\mathrm{p}}$ highlighting the minimum values of $E r r_{\mathrm{p}}$ by colour. Figure 1 illustrates Table 1 in a convenient chart form, which demonstrates the effect of $N_{\mathrm{p}}$ on the model efficiency - smaller bars indicate better efficiency.
The error norm Err defined by Eq. (4) and required for Eq. (6) was calculated twice for each formulation of model. For the models with small number of parameters $\left(N_{\mathrm{p}} \leq 5\right)$, a normalised $E r r$ produced better fitting, while for the models with many parameters $\left(N_{\mathrm{p}}>5\right)$, a non-normalised $\mathrm{Err}$ was better. Hence, a smaller value of Err was considered for each model in the assessment in Table 1 and Fig. 11 As a result, the following hyperelastic models ranking in order of their efficiency using the parametric error criterion (6) was produced with corresponding constants for Treloar's data22:

1. Response Function (RF) model ${ }^{1}$ with $E r r_{\mathrm{p}}=0.184$.

2. Extended Tube (ET) model $\frac{12}{12}$ with $\operatorname{Err}_{\mathrm{p}}=0.686$ and parameters: $G_{\mathrm{c}}=0.19232455[\mathrm{MPa}], G_{\mathrm{e}}=$ $0.19235307[\mathrm{MPa}], \beta=0.25976733, \delta=0.095741511$.

3. 4th order of Polynomial Form (PF) model $^{7}$ with $E r r_{\mathrm{p}}=$ 1.166 and parameters: $C_{10}=1.659 \cdot 10^{-1}, C_{01}=2.147$. $10^{-2}, C_{20}=-4.511 \cdot 10^{-3}, C_{11}=5.276 \cdot 10^{-4}, C_{02}=$ $-2.241 \cdot 10^{-4}, C_{30}=6.504 \cdot 10^{-4}, C_{21}=-1.031 \cdot 10^{-3}$, $C_{12}=9.082 \cdot 10^{-4}, C_{03}=-3.074 \cdot 10^{-4}, C_{40}=8.674$. $10^{-6}, C_{31}=-8.553 \cdot 10^{-5}, C_{22}=7.326 \cdot 10^{-5}, C_{13}=$ $2.273 \cdot 10^{-7}, C_{04}=-5.564 \cdot 10^{-9}[\mathrm{MPa}]$. 


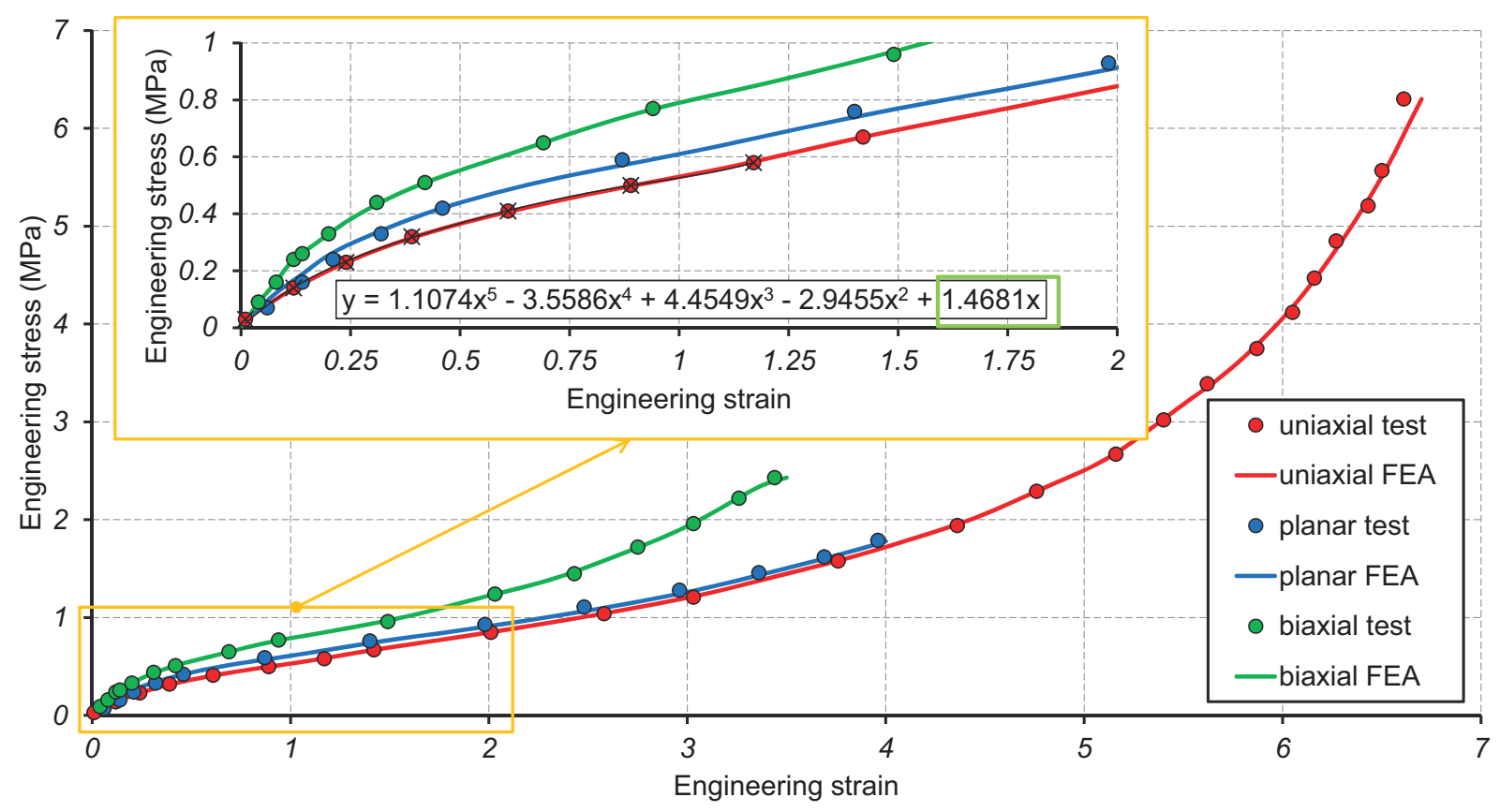

Fig. 2. Comparison of FE-simulations of the basic hyperelastic tests using the Response Function model $\frac{1}{\underline{1}}$ with Treloar's experiments 22

4. 5th order of Ogden model $\stackrel{8}{8}$ with $\operatorname{Err}_{\mathrm{p}}=1.366$ and parameters: $\mu_{1}=-6.23 \cdot 10^{7}[\mathrm{MPa}], \alpha_{1}=3.991 \cdot 10^{-3}$, $\mu_{2}=1.80355 \cdot 10^{-21}[\mathrm{MPa}], \alpha_{2}=24.6764 \cdot 10^{1}, \mu_{3}=$ $2.3324 \cdot 10^{-3}[\mathrm{MPa}], \alpha_{3}=4.67, \mu_{4}=2.235 \cdot 10^{7}[\mathrm{MPa}]$, $\alpha_{4}=6.29735 \cdot 10^{-3}, \mu_{5}=1.0854 \cdot 10^{8}[\mathrm{MPa}], \alpha_{5}=$ $9.94115 \cdot 10^{-4}$.

5. Gent model 10 with $E r r_{\mathrm{p}}=2.161$ and parameters $\mu=$ $0.273[\mathrm{MPa}], J_{\mathrm{m}}=84.623$ and Arruda-Boyce (AB) model $^{9}$ with $E r r_{\mathrm{p}}=2.499$ and parameters $\mu=$ $0.269[\mathrm{MPa}], \lambda_{\mathrm{L}}=4.635$.

6. 9-parameters form of Mooney-Rivlin (M-R) model 5.6 and 3rd order of PF model ${ }^{7}$ with $E r r_{\mathrm{p}}=2.544$ and parameters: $C_{10}=1.7225 \cdot 10^{-1}, C_{01}=9.5227 \cdot 10^{-3}$, $C_{20}=-1.9484 \cdot 10^{-3}, C_{11}=3.4357 \cdot 10^{-4}, C_{02}=$ $-1.2422 \cdot 10^{-4}, C_{30}=4.6579 \cdot 10^{-5}, C_{21}=5.2889$. $10^{-8}, C_{12}=3.56 \cdot 10^{-6}, C_{03}=-1.2791 \cdot 10^{-7}[\mathrm{MPa}]$.

\section{Overview of the efficient models}

In this ranking all the material parameters for the first 6 places were obtained using the non-normalised form of SSE (4). The ranking recommends the models, which are quite different from the ANSYS recommendations $\frac{36}{36}$ above. However, this ranking complies well with previous comparative studies and corresponding rankings $26,28,31,33,34$. The RF model taking the 1st place was denoted in the ranking of ANSYS models $\frac{31}{1}$ as the most effective model, which is able to fit experimental data in the complete range and not requiring material parameters identification. According to Dimitrov 31 , the RF model uses experimental data to determine the derivative of the hyperelastic potential with respect to the three principal invariants (constitutive response functions). Based on this information further, the material tangent matrix (second derivative of the hyperelastic potential) is calculated and used in the construction of the element incremental stiffness matrices. Therefore, the RF model is different from other hyperelastic models, formulated using strain energy function $W$, since it is a kind of computational model rather than a material model. Due to a not possible direct comparison of the RF model to experimental data in the ANSYS Curve Fitting Tool, the numerical results for uniaxial, biaxial, planar tests were obtained using FE-simulations with 3D models of specimens using SOLID285 FEs. Since the RF model doesn't contain any explicit material parameters, it matches ideally the experimental data 22 as shown in Fig. 2. Its only disadvantage is that it is not valid for extrapolation, since out of the experimental range the RF model produces purely elastic flow with zero stiffness $E=0$.

It should be noted that the error norm Err evaluation for the RF model was done in different way than for the rest of the models in ANSYS Curve Fitting Tool. The results of FE-simulations for each of the test (uniaxial, biaxial and 


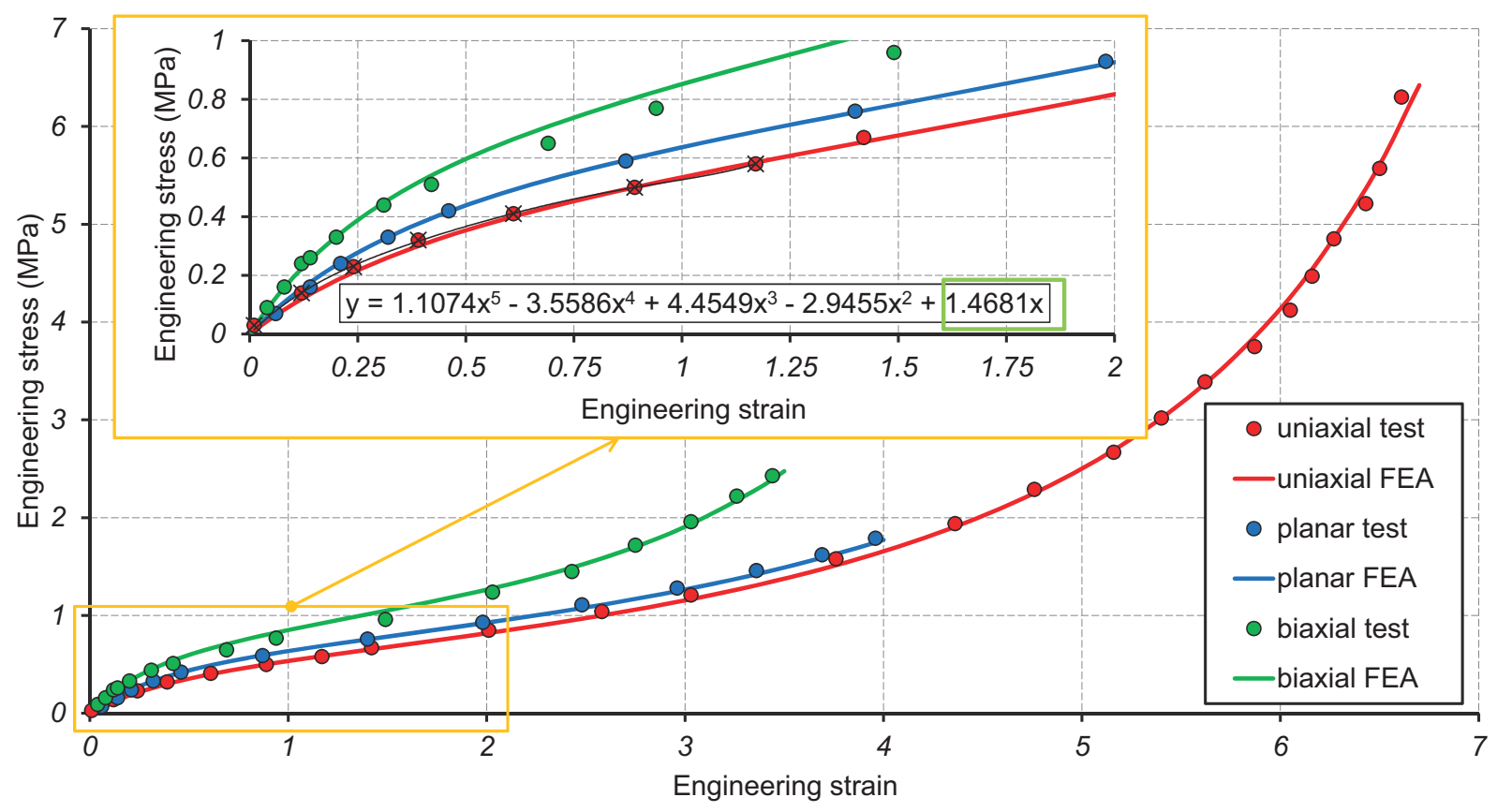

Fig. 3. Comparison of FE-simulations of the basic hyperelastic tests using the Extended Tube model ${ }^{12}$ with Treloar's experiments 22

planar) were fitted by polynomials of the 6th order in Excel:

$$
\begin{aligned}
\sigma_{\mathrm{u}}= & -6.88038 \cdot 10^{-5} \cdot \varepsilon^{6}+4.46440 \cdot 10^{-3} \cdot \varepsilon^{5} \\
& -5.47669 \cdot 10^{-2} \cdot \varepsilon^{4}+2.89142 \cdot 10^{-1} \cdot \varepsilon^{3} \\
& -6.94797 \cdot 10^{-1} \cdot \varepsilon^{2}+1.01823 \cdot \varepsilon, \\
\sigma_{\mathrm{b}}= & -2.86815 \cdot 10^{-2} \cdot \varepsilon^{6}+3.15550 \cdot 10^{-1} \cdot \varepsilon^{5} \\
& -1.35293 \cdot \varepsilon^{4}+2.90999 \cdot \varepsilon^{3} \\
& -3.26396 \cdot \varepsilon^{2}+2.19104 \cdot \varepsilon, \\
\sigma_{\mathrm{p}}= & -8.94005 \cdot 10^{-3} \cdot \varepsilon^{6}+1.17005 \cdot 10^{-1} \cdot \varepsilon^{5} \\
& -5.96681 \cdot 10^{-1} \cdot \varepsilon^{4}+1.51540 \cdot \varepsilon^{3} \\
& -1.99955 \cdot \varepsilon^{2}+1.58411 \cdot \varepsilon .
\end{aligned}
$$

These polynomial functions $\sigma_{\mathrm{u}}(\varepsilon), \sigma_{\mathrm{b}}(\varepsilon)$ and $\sigma_{\mathrm{p}}(\varepsilon)$ then were calculated at the same strain $\varepsilon$ values as experimental curves and compared to experimental stress $\sigma$ values. Thus, the normalised error norms Err were calculated for each curve using Eq. (4) and then summed up as $E r r_{\mathrm{p}}=0.184$ producing the results reported in Table 1 and Fig. 1

The second place is given to the ET model, which took the first place in rankings $26,31,33,34$. Referring to Dimitrov 31 , from the hyperelastic models requiring determination of material parameters the best one is the ET model because it involves only four parameters and its derivation is physically-motivated. Thus, the ET model matches the experimental data 22 almost perfectly as shown in Fig. 3 . Moreover, it is valid for extrapolation, since out of the experimental range the ET model keeps the realistic slope of the stress-strain curve.

An important feature of the ET model is that it is very sensitive to the initial values of parameters used as an input for fitting analysis when compared to all other models. Therefore, in most cases only a good guess of parameters, which all are $0<\operatorname{par}<1$, can guarantee a successful fitting result. There are several sets of material parameters for the ET model available in literature $\frac{26.34}{}$ to fit the Treloar's $\mathrm{data}^{22}$. The very first one is the original set after Kaliske \& Heinrich 12 (developers of the ET model), which gives the following values: $G_{\mathrm{c}}=0.1867[\mathrm{MPa}], G_{\mathrm{e}}=0.2169[\mathrm{MPa}]$, $\beta=0.2$ and $\delta=0.09693$. Therefore, this set of particular values was used as the initial values of hyperelastic parameters for fitting analysis. It was found that it provides a successful fitting with the ET model not only for the Treloar's data 22 in Fig. 3, but for a number of the experimental sets for rubbers-like materials reported in ${ }^{2}$ including:

- vulcanised natural rubber from ANSYS Mechanical APDL Technology Demonstration Guide $\underline{40}$,

- filled natural rubber after Mars and Fatemi ${ }^{41}$,

- cured natural rubber types EDS 19,16,15 and 14 after Gough et al. .42 , 

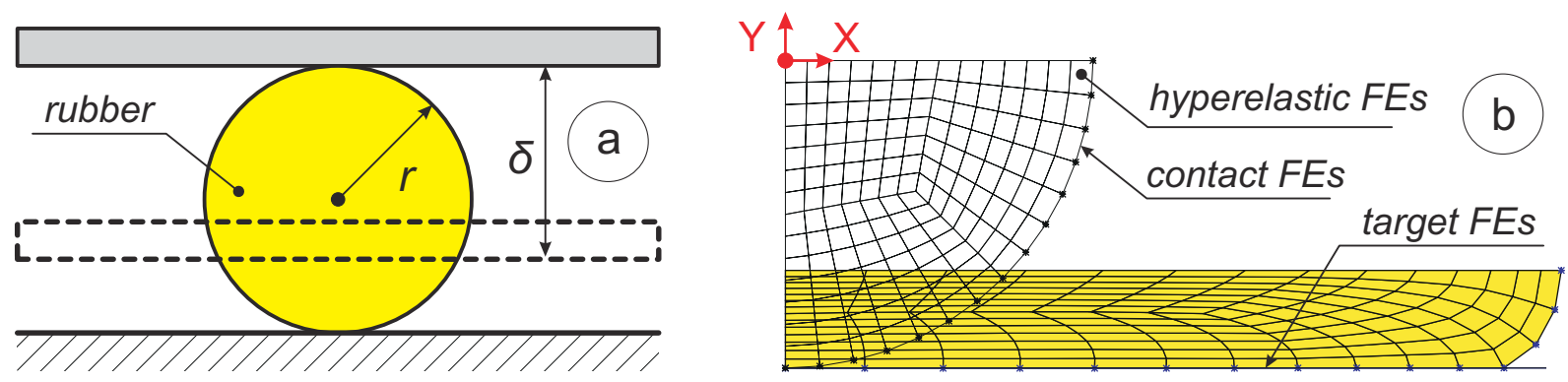

Fig. 4. Rubber cylinder benchmark: a) problem sketch and parameters, b) representative FE-model and deformed result

- $\quad$ synthetic rubbers (polyurethane, butyl, neoprene, viton, silicon, santoprene, hypalon) from SolidWorks material database $\mathrm{43}^{3}$.

The third place in ranking is given to the 4 rd order formulation of the Polynomial Form (PF) model ${ }^{7}$, which has the best fitting ability among all phenomenological models. It is based on both first $\bar{I}_{1}$ and second $\bar{I}_{2}$ strain invariants, and presents the most general mathematical formulation including all terms, when compared to other phenomenological models. According to Dimitrov 31 , this group of constitutive formulations is derived based on macromechanics of deformation. Specific is that material parameters are generally difficult to determine, and the phenomenological models have their deficits when used out of the deformation range in which their parameters were identified. Nevertheless, high order formulations of the PF model appear to have a very good fitting efficiency in the range of experimental data availability.

The fourth place in ranking is given to the 5 th order Ogden model ${ }^{8}$, another phenomenological model, which is in contrast directly based on the principal stretch ratios $\bar{\lambda}_{n}$ rather than the strain invariants $\bar{I}_{n}$. Since it is based on $\bar{\lambda}_{n}$ directly, it is capable of providing better data fitting. In general, Ogden model may be applicable for strains up to $700 \%$, but it is more computationally expensive than the rest of the models. Ogden model also took the fourth place in the ranking by Marckmann \& Verron ${ }^{26}$, the highest place among conventional phenomenological models in that study.

The fifth place in ranking is given to Gent $\frac{10}{}$ and $A-B=9$ models, which both belong to the group of micro-mechanical models. According to Dimitrov $\stackrel{31}{ }$, the models of this group are derived based on careful study of stochastic kinetics of deforming polymer chains. Such models lead to hyperelastic potentials depending on micro-mechanical deformation mechanisms observed in the elastomer. The A-B model, also known as the eight-chain model, is a statistical mechanicsbased model. This means that its form was developed as a statistical treatment of non-Gaussian chains emanating from the centre of the element to its corners. A-B model and similar Gent model, both having only 2 material parameters, appear to be quite effective due to an advanced background and sophisticated mathematical form.

The sixth place in ranking is given to the 9-parameters formulation of the M-R model ${ }^{5.6}$, which is similar to the $3 \mathrm{rd}$ order formulation of the PF model ${ }^{7}$. This model was historically one of the first hyperelastic models, and also belongs to the group of phenomenological models. It is based on the observation that rubber response is linear under simple shear loading conditions. The 9-parameters M-R model was denoted as the best in the comparative study by Ruíz \& González $\underline{28}$ for application to elastomeric fabrics. Despite of an old mathematical formulation, this model proves to be effective as well.

\section{Modification of the benchmark problem}

\section{Purpose of benchmark problems}

The concept of benchmarks is widely used in computational mechanics and particularly for modelling of nonlinear material behaviour. The reference solutions of benchmark problems are usually presented by analytic or semianalytic solutions called design equations. In case of elastomeric structures, they are available for a number of simple shapes $\frac{18}{}$. For each geometry considered, the equation produces the stiffness, the force per unit displacement, or the force per unit length or width. Referring to Bauman $\frac{18}{}$ there are several circumstances when design equations are useful:

- FE-code is expensive to lease and engineers proficient in its use are not readily available;

- a feasibility study is required, so formulas are adequate; 
- only simple shapes described by the formulas are used;

- the part is not structurally critical;

- stress-strain data required to determine the coefficients for the constitutive law for FEA are not available.

In this study, the benchmark problem is used for the basic verification of the hyperelastic material input by comparing the FEA solution to a corresponding reference solution.

The subdivision into two broad categories of formulas is proposed by Bauman $\stackrel{18}{ }$. The first set consists of the traditional ones that depend on small rubber deformations (typically $<30 \%$ ), approximately linear rubber stressstrain behaviour, and incompressible material. These equations have been studied and systematised by Lindley 44 and Gent ${ }^{45}$. The second category, developed by Yeoh, Pinter and Banks 46 applies to larger strains, allows for slight compressibility and approximates FEA solutions for some simple shapes. However, this study presents a modification of the reference solution for a conventional benchmark from the first category, which extends its applicability to large strains of about $150 \%$.

\section{Compression of rubber cylinder}

Referring to Lindley 44 , when a curved surface of a rubber component is compressed against a rigid plane, the stiffness generally increases as the area of contact increases during the deformation. Thus, the load-deformation characteristics tend to be markedly non-linear. For the rollers (solid, hollow and rubber-covered) the relationships apply for plane strain conditions, i.e. for length $\gg$ rubber thickness.

This conventional benchmark problem for elastomers is comprehensively studied by Sussman and Bathe $\underline{47}$ using a displacement-pressure ( $\mathrm{u} / \mathrm{p})$ FE-formulation for the geometrically and materially nonlinear analysis of compressible and almost incompressible solids. One of the study objectives $\underline{47}$ was a determination of the force-deflection curve for the cylinder and also the location and magnitude of the maximum stresses when the applied displacement equals one-half of the initial diameter of the cylinder. The geometry is defined in Fig. 4 $4 \mathrm{a}$ and shows $r$ as the outside radius of the roller and $\delta$ as the compressive displacement.

For small displacements, the Hertz contact assumptions are valid, and the following force per unit length $(f)$ vs. deflection $(\delta)$ relationship $\stackrel{48}{\underline{4}}$ results in:

$$
\begin{aligned}
& \delta=\frac{4 f}{\pi} \frac{1-v^{2}}{E_{0}}\left(\frac{1}{3}+\ln \left[\frac{4 r}{b}\right]\right), \\
& \text { with } b=1.6 \sqrt{2 r f \frac{1-v^{2}}{E_{0}}},
\end{aligned}
$$

where $E_{0}$ and $v$ are the small strain Young's modulus and Poisson's ratio correspondingly.

For larger displacements during compression of solid rubber rollers, an approximate solution based on experiments is given by Lindley $\underline{44}$ for the force per unit length as follows

$$
\frac{f}{6 r G}=1.25\left(\frac{\delta}{2 r}\right)^{1.5}+50\left(\frac{\delta}{2 r}\right)^{6},
$$

which doesn't account for the effect of friction. Using incompressibility assumption $v \approx 0.5$ in formula for shear modulus

$$
G=\frac{\tau}{\gamma}=\frac{E}{2(1+v)}
$$

the Eq. (11) is simplified to the following form:

$$
f=E_{0} r\left[2.5\left(\frac{\delta}{2 r}\right)^{1.5}+100\left(\frac{\delta}{2 r}\right)^{6}\right],
$$

where the Young's modulus $E_{0}$ is assumed to be constant.

In order to assess the accuracy of the available analytic solutions presented by Eqs (10) and (13), a sample benchmark case has been analysed numerically in the FE-code ANSYS. This case is based upon the sample benchmark formulation used by Sussman \& Bathe $\frac{47}{7}$ for FE-code ADINA shown in Fig. 4h, which comprised:

- cylinder radius of $r=200 \mathrm{~mm}$;

- plane strain consideration with infinite cylinder length;

- frictionless contact using node-to-surface contact type;

- maximum displacement of the plate as $\delta=200 \mathrm{~mm}$.

The objective is to determine the force-deflection response using FEA and compare it to the reference solutions (10) and (13). Due to geometric and loading symmetry, the FE-analysis is performed using one quarter of the cyclinder cross section with 14 FEs per radius as shown in Fig. 4b. All nodes on the left edge $(X=0)$ are constrained in $U X$. All nodes on the top edge $(Y=0)$ are coupled in $U Y$. An imposed displacement of $\delta / 2$ acts upon the coupled nodes. The quasistatic problem is solved using the $2 \mathrm{D}$ lower order 


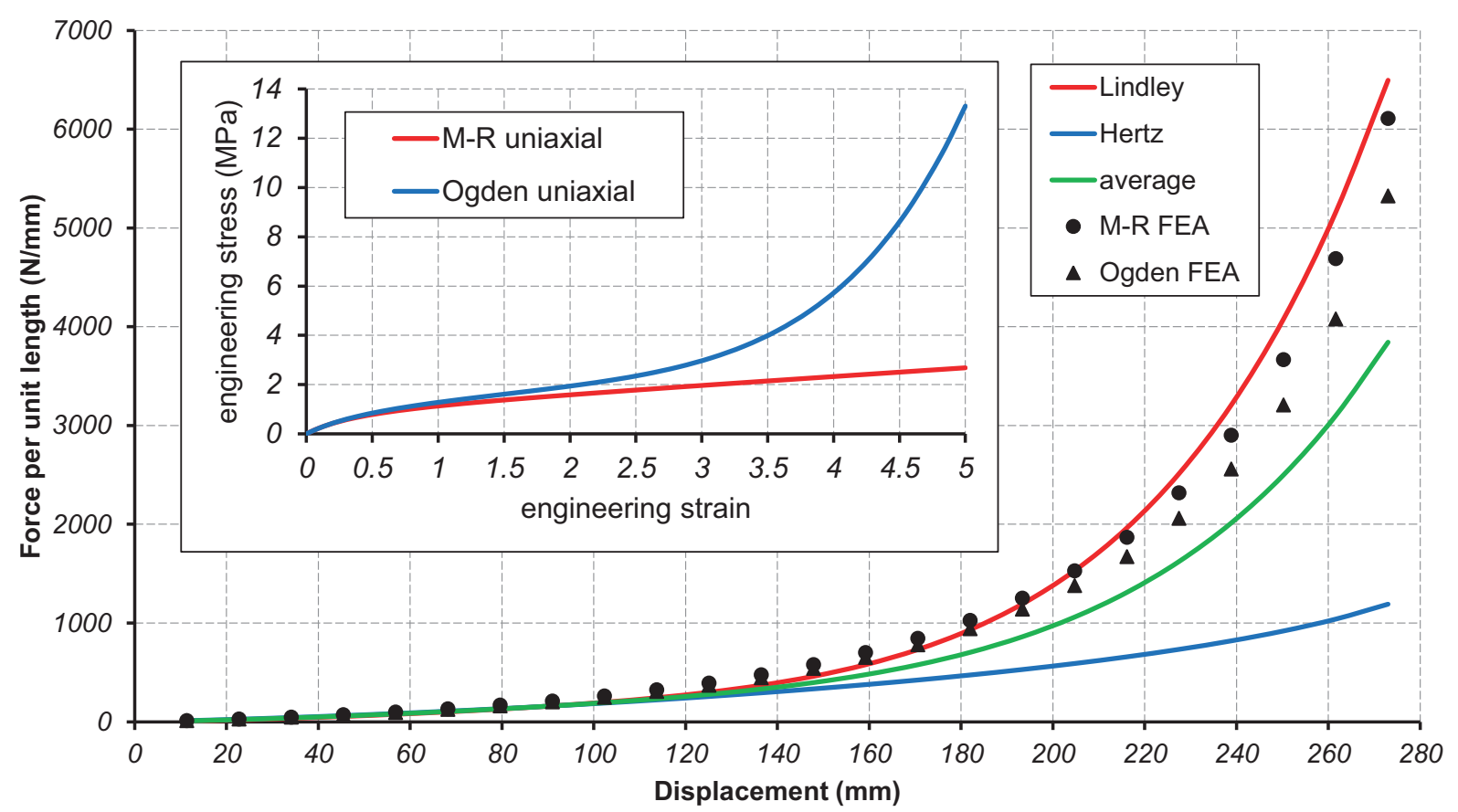

Fig. 5. Comparison of analytical solutions, Lindley's Eq. [13, Hertz's Eq. 10] and their average Eq. 15, to FEA results obtained in ANSYS using Mooney-Rivlin 5,6 and Ogden $\frac{8}{}$ material models

solid elements (PLANE182), rigid target (type TARGE169) and contact (CONTA175) elements. The solution is obtained in a number of substeps using large deformations assumption and default contact algorithm.

\section{Modification of the reference solution}

There are a few improvements in the formulation of the current benchmark when compared to the previous one $e^{47}$ as explained below. Firstly, The maximum imposed displacement is increased from original $\delta=200$ to $273 \mathrm{~mm}$, which corresponds to $\varepsilon_{\mathrm{t}}=150 \%$ of equivalent true strain in structure or $\varepsilon_{\mathrm{e}}=350 \%$ of equivalent engineering strain on the stress-strain curve.

Secondly, the Treloar's experimental data set $\underline{22}$ is used in this study instead of hyperelastic model fits $\frac{47}{}$ based upon the 3-terms form of Mooney-Rivlin model ${ }^{5.6}$ and the 3rd order of Ogden model ${ }^{8}$. The corresponding solution of the benchmark problem, previously obtained in ADINA ${ }^{47}$, was derived in ANSYS as illustrated in Fig.5. It should be noted that for displacements up to $\delta=200 \mathrm{~mm}$, FE-results with both material models are quite close to Lindley's Eq. (13) as obtained by Sussman \& Bathe ${ }^{47}$. However, for the larger displacements up to $\delta=273 \mathrm{~mm}$, the FE-result with the Ogden model deviates from Lindley's solution, while FE-result with
M-R model keeps close to it. Moreover, the material fit using 6-terms Ogden model is more accurate than the fit with 3-terms M-R model. This fact reveals that the Lindley's solution (13) is non-conservative for large displacements and significant compression of the cylinder, since the FE-result with Ogden model is more realistic. The stress-strain curve of the M-R fit provides a much softer material response than the more advanced Ogden fit.

Since the experimental data for the rubber in the original benchmark is unavailable, the hyperelastic model fits $\underline{47}$ have been replaced with the most accurate Response Function model $^{1}$ fit of the Treloar's data ${ }^{22}$. The RF model doesn't need any curve fitting procedures, so it is used directly in FE-simulation of the benchmark problem by attaching available experimental stress-strain curves. The obtained FE-results shown in Fig. 6 appear to be in between two conventional analytical solution. The Lindley's Eq. (13) is an upper bound providing a non-conservative prediction for softer elastomers, while the Hertz's Eq. (10) is a lower bound providing a conservative prediction for harder elastomers.

This yields into the third improvement been proposed, which is related to the accuracy of the analytic predictions in the benchmark problem. Based upon the obtained FEresults for the benchmark, an average of two conventional analytical solutions (13) and (10) is proposed. The problem 


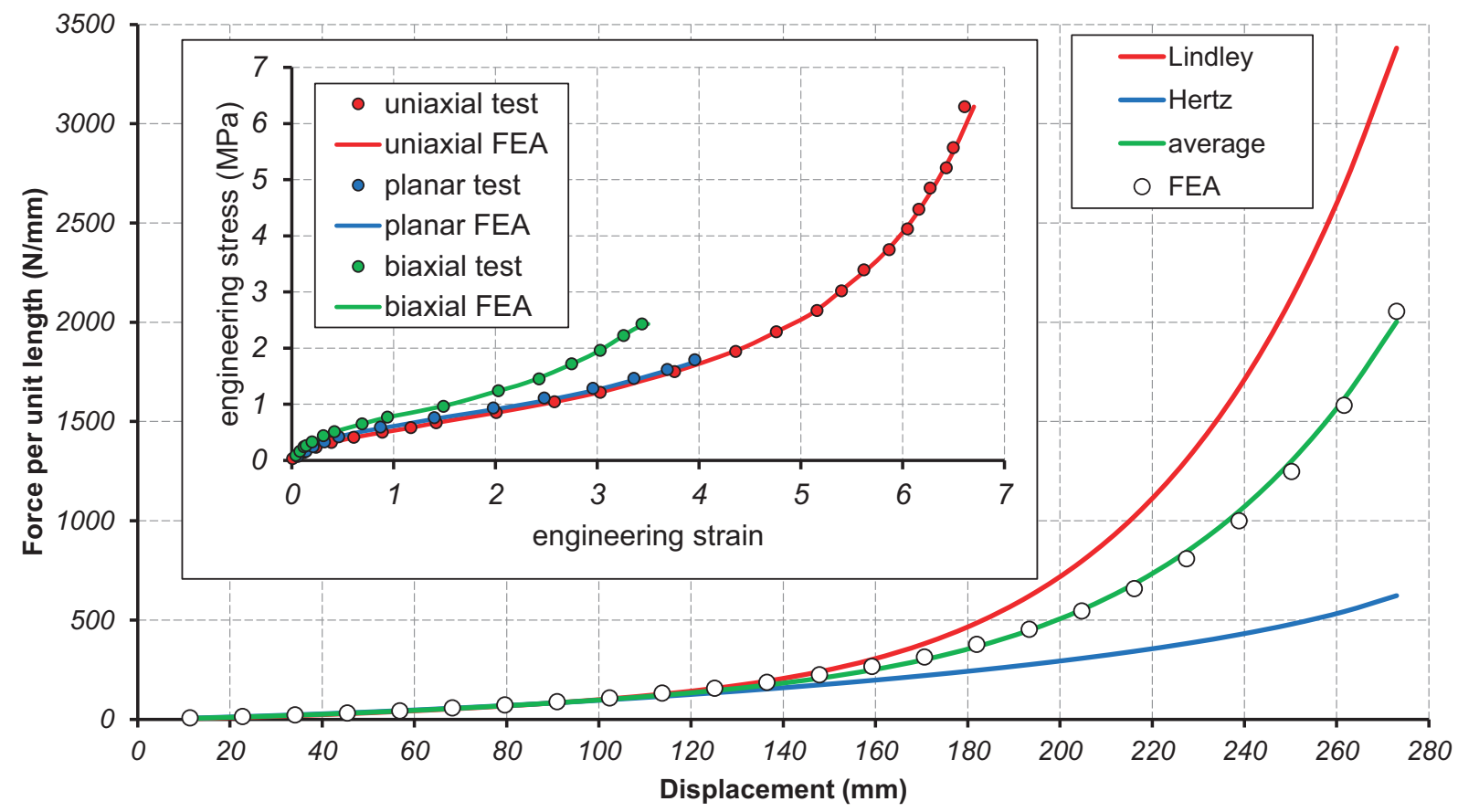

Fig. 6. Comparison of analytical solutions, Lindley's Eq. [13, Hertz's Eq. 10] and their average Eq. [15, to FEA results obtained in ANSYS using the Response Function material model 1

of this combination is that the Lindley's solution is given as force dependent on displacement $f(\boldsymbol{\delta})$, while the Hertz's solution is given as displacement dependent on force $\delta(f)$. Since they are not dependent on one variable, one of them needs to be reversed mathematically to become compatible for their combination. The direct mathematical reversion is problematic for both of the formulas (13) and (10), since the dependent variables are presented twice in both of them within the power-law functions with different power exponents. Thus, a non-direct recursive approach is applied here to reverse the function $\delta(f)$ in $(10)$ as

$$
f_{n+1}=\frac{\delta \frac{\pi}{4} \frac{E_{0}}{1-v^{2}}}{\frac{1}{3}+\ln \left(\frac{4 r}{1.6 \sqrt{2 r f_{n} \frac{1-v^{2}}{E_{0}}}}\right)}
$$

where $n \geq 10$ and the initial iteration $f_{0}$ is defined by (13). This recursive approach is similar to the one used by Gorash \& Chen ${ }^{49.50}$ to reverse the formula for bending moment dependent on total strain, which is applied to a beam with a square cross-section to deform it plastically using the Ramberg-Osgood material model.
Then a simple averaging is applied to Eqs (13) and (14)

$$
f^{\mathrm{ave}}(\delta)=\frac{f_{n+1}^{H}(\delta)+f^{L}(\delta)}{2},
$$

where $f_{n+1}^{H}(\delta)$ is the Hertz's Eq. (14) in the reversed form and $f^{L}(\delta)$ is the Lindley's Eq. (13).

The average solution (15) of the benchmark problem using Treloar's data ${ }^{22}$ illustrated in Fig. 6. matches perfectly the FE-results obtained with the RF model ${ }^{1}$, which is the most accurate compared to other hyperelastic models. Thus, an introduction of the average solution using Eqs (14) and (15) extends the applicability of the benchmark problem to large displacements. The conventional Lindley's solution (13), limited to about $50 \%$ of true strain, becomes valid for about $150 \%$ of true strain in combination with the reversed Hertz's solution (14).

It should be noted that the analytical benchmark input requires only one material parameter $-E_{0}$, elasticity modulus or initial slope of the hyperelastic stress-strain curve. It is defined by application of the trendline in Excel to the initial range of the uniaxial stress-strain curve. The regression type of the trendline is usually a polynomial of the 5 th or 6 th order, which intercepts the coordinates origin $[0,0]$. Therefore, the coefficient of the 1st order component represents $E_{0}$, since it is the only non-zero number of the polynomial 
derivative defined in the location $[0,0]$. An example of the $E_{0}$ estimation is illustrated in Figs 2 and 3 where the 5 th order polynomial is applied to the strain range of $[0,1.17]$ of uniaxial curve, and correspondent $E_{0}=1.468 \mathrm{MPa}$.

\section{Benchmark applied to other elastomers}

Apart from Treloar's data 22 , the benchmark was applied to other natural and synthetic rubbers investigated in report $\stackrel{2}{2}$. Each set of stress-strain curves $\stackrel{2}{2}$ has a polynomial trendline attached with a correspondent equation, last component of which represents $E_{0}$. Numerical solutions of the benchmark were derived with the Response Function model used to fit stress-strain curves, while analytical solutions (13), (10) and (15) were obtained using a correspondent value of $E_{0}$. All the range of FE-solutions is located between Lindley's (13) and Hertz's (10) solutions. Harder rubbers with a steeper initial slope are closer to the Hertz's (10) solution, while softer rubbers with less steep initial slope are closer to the Lindley's (13) solution. The full classification of material response according to the numerical benchmark response is following:

Pure soft: Neoprene and butyl rubbers $\frac{43}{3}$;

Soft-average: Vulcanised natural rubber ${ }^{40}$ and hypalon rubber $\stackrel{43}{ }$;

Pure average: Filled natural rubber $\stackrel{41}{ }$, rubber EDS $19 \underline{42}$ and polyurethane $\stackrel{43}{ }$;

Hard-average: Rubber EDS $14 \stackrel{42}{ }$, silicon rubber and viton fluoroelastomer $\stackrel{43}{ }$;

Pure hard: Santoprene $\stackrel{43}{ }$;

Mixed (initially hard): Rubbers EDS 16 and $15 \underline{42}$.

It should be noticed that the proposed benchmark enables verification of material model fits for a wide number of elastomers, which are quite different in the shape of experimental stress-strain curves. Since the numerical solution for the majority of the tested elastomers tends to the average analytical solution (15), the proposed modification of the benchmark proves to be quite significant.

\section{Functionality of the developed CAE-based application}

Fitting of test stress-strain curves by hyperelastic models and verification of obtained material parameters by the solution of an improved benchmark problem, which are described in previous sections, are implemented in a standalone Windows-application. This application was developed using Visual Basic .NET language in Microsoft Visual Studio 2010 environment. Different inter-process communication mechanisms are used in interactions with several external applications. The most important component of its functionality is an implementation of a two-way interaction with ANSYS as a single loop using the APDL-script as an input and text, graphical and video files as an output. Each time when the analysis is run in the application, the following 3 -steps procedure is executed:

- generation of the input APDL-script in a text file according to the above defined options;

- starting of ANSYS executable file in batch mode, which reads and executes the input APDL-script;

- text, graphical and video files generated by ANSYS are uploaded into the application for review.

The Windows API functionality is used by the application to start ANSYS executable file as a process and to wait until it is completed. The hyperelastic identification module of the application has the following structure as shown in Fig. 7

1. ComboBox with choice of available experimental data for a number of elastomers considered in this work ${ }^{2}$.

2. ComboBox with choice of isotropic hyperelastic material models supported by ANSYS $^{\underline{1}}$ as indicated in Introduction.

3. TextBox with a small strain (initial) elasticity modulus $E_{0}$ identified using a uniaxial stress-strain curve in Excel as explained in the Benchmark Modification. It is a part of the data set provided by a user along with stress-strain curve in separate text files.

4. Options for a hyperelastic model formulation comprising a choice of polynomial order or number of constants.

5. CheckBox for a choice of normalised or non-normalised error norm used in fitting analysis as explained in the Least Squares Fit Analysis.

6. TextBox defines a maximum number of iterations in fitting analysis governing the accuracy and duration of analysis. 


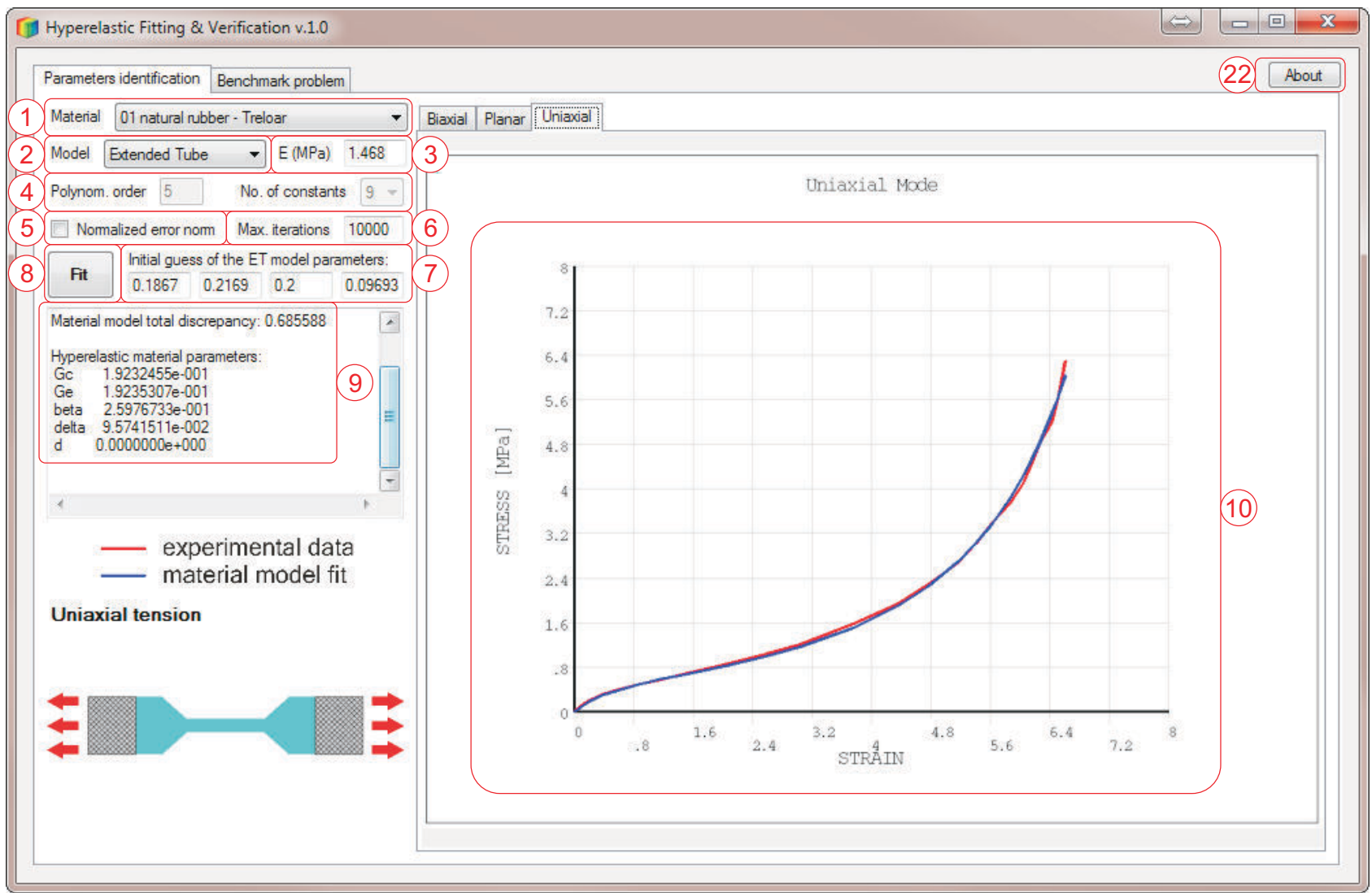

Fig. 7. Structure of GUI of the CAE-based application - interface for fitting of hyperelastic material parameters

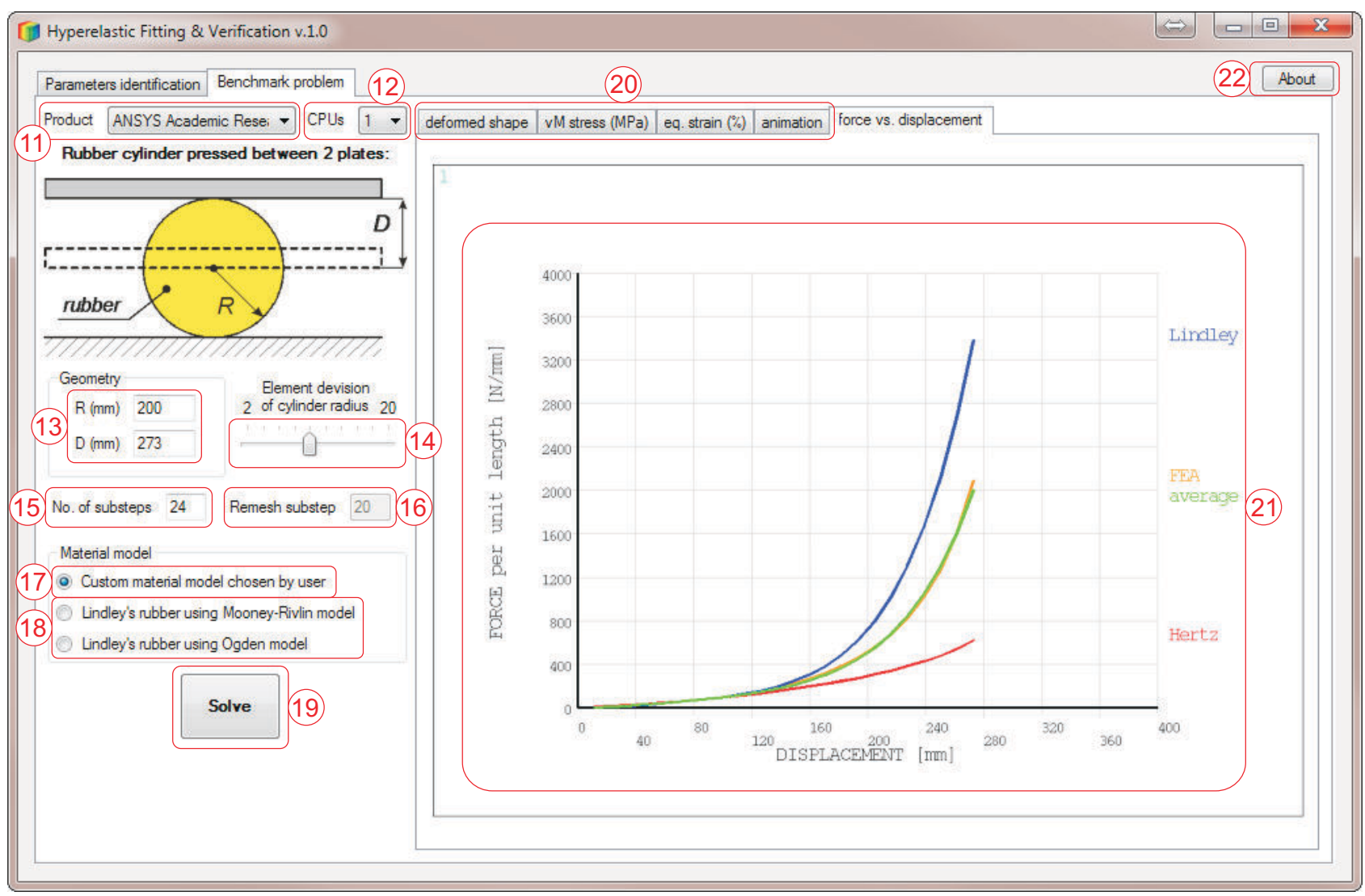

Fig. 8. Structure of GUI of the CAE-based application - interface for setting up of the benchmark and viewing results 
7. TextBoxes with a mandatory initial guess of the ET model ${ }^{12}$ parameters, which is explained in the Overview of the Efficient Models.

8. Button for running of the fitting analysis in ANSYS.

9. TextBox for fitting analysis output in text form:

- $\quad$ error norm or SSE defined by Eq. (4);

- number of non-zero parameters in a material model;

- $\quad$ fitting quality criterion, called parametric error, which is explained in the Fitting Quality Criteria and defined by Eq. (6).

10. TabPages for fitting analysis output in graphical form, which display the comparisons of material model fit to experimental data for all available stress-strain curves.

The hyperelastic verification module of the application has the following structure as shown in Fig. 8 .

11. ComboBox with choice of license for ANSYS product. 12. ComboBox with number of CPUs requested for FEA.

13. TextBoxes define variable parameters $(r$ and $\delta$ ) of the benchmark geometry as discussed in the Compression of Rubber Cylinder.

14. TrackBar governs the default size of finite elements by specifying the number of radius divisions for the FE-mesh.

15. TextBox defines a number of substeps in non-linear FE-analysis of the benchmark as discussed in the Compression of Rubber Cylinder.

16. TextBox (currently suppressed) defines a substep, when the remeshing is required due to excessive FE distortion. This option will be implemented in the next version.

17. RadioButton specifies the application of a custom material model chosen by a user for the benchmark FEanalysis.

18. RadioButtons specify the application of conventional hyperelastic models and corresponding material constants $\frac{47}{}$ as explained and illustrated in the Benchmark Modification. This option is implemented for testing purpose.

19. Button for running of the benchmark FEA in ANSYS.

20. TabPages for FEA output in graphical form including:

- final deformed shape of the FE-mesh,

- contours of equivalent von Mises stress and strain,

- $\quad$ animation of the cylinder deformation over time.
21. TabPage with diagram of force vs. deflection response, which allows to compare FEA result with 3 reference solutions as explained in the Benchmark Modification and conclude about the hyperelastic model suitability as explained in the Application to Other Elastomers.

Button "About" highlighted as item no. 22 in Figs 7 and 8 contains the technical information regarding application development. FEA results for the deformed shape and contours of equivalent von Mises stress and strain are output by ANSYS in 3D form using VRML files. These files are uploaded into the application immediately after the FEA execution and run for viewing in corresponding TabPages shown as position 9 in Fig. 8. Viewing of the VRML files is implemented by the integrated graphical components of Cortona3D Viewer, which typically works as a VRML plugin for popular Internet browsers and Microsoft Office applications on Windows platform, and it needs to be installed before running the application. The animation of the cylinder deformation over time in AVI file is implemented by the integrated graphical component of Windows Media Player, which is used for playing audio, video and viewing images on Windows platform. The AVI file is produced from the sequence of JPEG files generated by ANSYS at every FEA substep using FFmpeg, which is a cross-platform and free software to record, convert and stream audio and video. The application interacts with FFmpeg executable file included in the installation folder in the same way as with ANSYS.

\section{Conclusions}

This paper presents a theoretical background on the CAEbased application for identification and verification of hyperelastic material parameters and an overview of its functionality. The most important outcomes of this study are:

- Ranking of hyperelastic models efficiency, which was estimated using a new fitting quality criterion.

- Recent hyperelastic models (Extended Tube and Response Function) were found as the most efficient.

- Modified reference solution for the classical benchmark, which made it valid for large deformation.

- Developed application interacting with ANSYS for the effective implementation of the study. 
Section "Introduction" presents an overview of the isotropic hyperelastic models supported by ANSYS and literature review on comparative studies, rankings and hyperelastic model assessments over the last years. Section "Assessment of hyperelastic models efficiency" includes curve fitting tools overview, basics of least squares fit analysis, formulation of the new fitting quality criteria, ranking of isotropic incompressible hyperelastic models supported by ANSYS, and analysis of the most efficient models. Section "Modification of the benchmark problem" includes explanation of benchmark problems purpose, formulation and FEA of a classical benchmark for rubber cylinder compression, proposed modification of the reference solution for this benchmark, and application of this benchmark to available experimental data for other elastomers. The last section presents the overview of the programming, structure and functionality of the developed CAE-based application. The wide applicability of the developed approach and CAEbased application has been confirmed using experimental stress-strains curves for 7 natural and 7 synthetic rubbers.

However, one important aspect of elastomeric components modelling has not been investigated in this work. It is the effect of friction on the force response of the O-ring, which has quite a significant contribution. In general, the friction between elastomers and solid materials is a complex phenomenon, where the coefficient of friction $\mu$ is dependent on the normal pressure in contact surface, e.g. in the power-law form as discussed by Wriggers $\frac{51}{1}$. The significant contribution of the friction on contact pressure compared to other factors in a seal mechanism has been experimen-

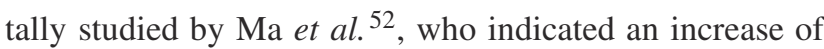
$\mu$ (e.g. from 0.3 to 0.7 ) with increase of external loading. Moreover, Lindley $\underline{53}$ also studied experimentally the effect of friction on the load-compression behaviour of an O-ring for different values of $\mu(0.02,0.1,0.7)$ and indicated a lift of load-deformation curve with increase of $\mu$. Equation (11) has also been extended by inclusion of $\mu$ providing an opportunity to study the effect of friction analytically. However, the numerical simulations with consideration of friction $(\mu$ $>0.1$ ) get obstructed by highly distorted elements for large displacement of the plate $\delta>100 \mathrm{~mm}$. In order to investigate the compression of O-ring with friction by FEA for the larger displacements up to $\delta=273 \mathrm{~mm}$, an application of adaptive remeshing technique in ANSYS is required. The initial study $\underline{54}$ of this relatively new numerical technique has recently been carried out by simulation of the extrusion of rubber O-rings through the gaps in flanges. The work on consideration of frictional contact with application of adaptive remeshing for the simulations of elastomeric components undergoing large deformation is currently in progress.

\section{Supplementary files}

This paper is supplemented with the above-discussed Windows-application "Hyperelastic fitting \& verification" in the form of ZIP-archive containing the following files:

- main executable file of the application titled "hyper_fit_n_verify.exe";

- 4 dynamic-link libraries (DLL) used for interaction with Cortona3D Viewer and Windows Media Player;

- folder with experimental stress-strain curves for the hyperelastic materials investigated in this work;

- text file "readme.txt" with short instruction related to prerequisites and proper running of this application.

\section{Acknowledgements}

The authors greatly appreciate the R\&D Elastomer Development department of Weir Minerals for the financial and material support in the frames of WARC project $\mathrm{C}_{2} \underline{2}$ and the University of Strathclyde for hosting during the course of this work.

\section{References}

[1] ANSYS ${ }^{\circledR}$ Academic Research. Help System // Mechanical APDL // Material Reference // 3. Material Models // 3.6. Hyperelasticity. Canonsburg (PA), USA; 2013.

[2] Gorash Y, Comlekci T, Hamilton R. Material modelling and numerical simulation of elastomeric components in pumps and valves. University of Strathclyde, Glasgow, UK: Weir Advanced Research Centre (WARC); 2014. Project C2.

[3] Macosko CW. Rheology: principles, measurement and applications. New York, USA: Wiley-VCH; 1994.

[4] Ogden RW. Nonlinear elastic deformations. New York, USA: Dover Publications; 1997.

[5] Mooney M. A theory of large elastic deformation. J Appl Phys. 1940;11(9):582-592. DOI: 10.1063/1.1712836.

[6] Rivlin RS. Large elastic deformations of isotropic materials. IV. Further developments of the general theory. Phil Trans R Soc Lond A. 1948;241(835):379-397. DOI: $10.1098 /$ rsta.1948.0024 
[7] Rivlin RS, Saunders DW. Large elastic deformations of isotropic materials. VII. Experiments on the deformation of rubber. Phil Trans R Soc Lond A. 1951;243(865):251-288. DOI: $10.1098 /$ rsta.1951.0004.

[8] Ogden RW. Large deformation isotropic elasticity: On the correlation of theory and experiment for compressible rubberlike solids. Proc R Soc Lond A. 1972;328(1575):567-583. DOI: $10.1098 / \mathrm{rspa}$.1972.0096.

[9] Arruda EM, Boyce MC. A three-dimensional constitutive model for the large stretch behavior of rubber elastic materials. J Mech \& Phys Solids. 1993;41(2):389-412. DOI: $10.1016 / 0022-5096(93) 90013-6$

[10] Gent AN. A new constitutive relation for rubber. Rubber Chem \& Technol. 1996;69(1):59-61. DOI: 10.5254/1.3538357

[11] Yeoh OH. Some forms of the strain energy function for rubber. Rubber Chem \& Technol. 1993;66(5):754-771. DOI: $10.5254 / 1.3538343$.

[12] Kaliske M, Heinrich G. An extended tube-model for rubber elasticity: Statistical-mechanical theory and finite element implementation. Rubber Chem \& Technol. 1999;72(4):602632. DOI: $10.5254 / 1.3538822$.

[13] Blatz PJ, Ko WL. Application of finite elastic theory to the deformation of rubbery materials. Trans Soc Rheol. 1962;6(1):223-252. DOI: 10.1122/1.548937

[14] Ogden RW. Recent advances in the phenomenological theory of rubber elasticity. Rubber Chem \& Technol. 1986;59(3):361383. DOI: $10.5254 / 1.3538206$.

[15] Marlow RS. A general first-invariant hyperelastic constitutive model. In: Busfield J, Muhr A, editors. Proc. 3rd Euro. Conf. on Constitutive Models for Rubber III. London, UK: Taylor \& Francis; 2003. p. 157-160.

[16] Sussman T, Bathe K -J. A model of incompressible isotropic hyperelastic material behavior using spline interpolations of tension-compression test data. Commun Numer Meth Engng. 2009;25(1):53-63. DOI: 10.1002/cnm.1105

[17] Gough J, Gregory IH, Muhr AH. The Uncertainty of Implemented Curve-Fitting Procedures in Finite Element Software. In: Boast D, Coveney VA, editors. Finite Element Analysis of Elastomers. London: Professional Engineering Publishing; 1999. p. 141-151.

[18] Bauman JT. Fatigue, Stress, and Strain of Rubber Components. Munich: Carl Hanser Verlag; 2008.

[19] Finney RH. Finite Element Analysis. In: Gent AN, editor. Engineering with Rubber: How to Design Rubber Components. 3rd ed. Munich: Carl Hanser Verlag; 2012. p. 295-343.
[20] Seibert DJ, Schöche N. Direct comparison of some recent rubber elasticity models. Rubber Chem \& Technol. 2000;73(2):366-384. DOI: 10.5254/1.3547597

[21] Boyce MC, Arruda EM. Constitutive models of rubber elasticity: A review. Rubber Chem \& Technol. 2000;73(3):504-523. DOI: $10.5254 / 1.3547602$.

[22] Treloar LRG. Stress-strain data for vulcanised rubber under various types of deformation. Trans Faraday Soc. 1944;40:59-70. DOI: $10.1039 /$ TF9444000059

[23] Xia Y, Li W, Xia Y. Study on the compressible hyperelastic constitutive model of tire rubber compounds under moderate finite deformation. Rubber Chem \& Technol. 2004;77(2):230-241. DOI: $10.5254 / 1.3547820$

[24] Chagnon G, Marckmann G, Verron E. A comparison of the HartSmith model with Arruda-Boyce and Gent formulations for rubber elasticity. Rubber Chem \& Technol. 2004;77(4):724-735. DOI: $10.5254 / 1.3547847$

[25] Attard MM, Hunt GW. Hyperelastic constitutive modeling under finite strain. Int J Solids \& Struct. 2004;41(18-19):5327_ 5350. DOI: 10.1016/j.ijsolstr.2004.03.016

[26] Marckmann G, Verron E. Comparison of hyperelastic models for rubber-like materials. Rubber Chem \& Technol. 2006;79(5):835-858. DOI: 10.5254/1.3547969

[27] Kawabata S, Matsuda M, Tei K, Kawai H. Experimental survey of the strain energy density function of isoprene rubber vulcanizate. Macromolecules. 1981;14(1):154-162. DOI: $10.1021 / \mathrm{ma} 50002 \mathrm{a} 032$

[28] Ruíz MJG, González LYS. Comparison of hyperelastic material models in the analysis of fabrics. Int J Clothing Sci \& Technol. 2006;18(5):314-325. DOI: 10.1108/09556220610685249

[29] Vahapoğlu V, Karadeniz S. Constitutive equations for isotropic rubber-like materials using phenomenological approach: A bibliography (1930-2003). Rubber Chem \& Technol. 2006;79(3):489-499. DOI: 10.5254/1.3547947

[30] Ali A, Hosseini M, Sahari BB. A review of constitutive models for rubber-like materials. Amer J Eng \& Appl Sci. 2010;3(1):232-239. DOI: 10.3844/ajeassp.2010.232.239

[31] Dimitrov S. Review of the basic hyperelastic constitutive models in ANSYS 13.0. CADFEM Infoplaner. 2011;01/2011:52-53. URL: WWW. cadfem.de/unternehmen/cadfem-journal/ [32] Li M, Hu X, Luo W, Huang Y, Bu J. Comparison of two hyperelastic models for carbon black filled rubber. Appl Mech \& Mater. 2013;275-277:28-32. DOI: $10.4028 / w w w . s c i e n t i f i c . n e t / A M M .275-277.28$ 
[33] Steinmann P, Hossain M, Possart G. Hyperelastic models for rubber-like materials: consistent tangent operators and suitability for Treloar's data. Arch Appl Mech. 2012;82(9):1183-1217. DOI: $10.1007 / \mathrm{s} 00419-012-0610-\mathrm{Z}$

[34] Hossain M, Steinmann P. More hyperelastic models for rubber-like materials: consistent tangent operators and comparative study. J Mech Behav Mater. 1998;22(1-2):27-50. DOI: $10.1515 / \mathrm{jmbm}-2012-0007$

[35] Beda T. An approach for hyperelastic model-building and parameters estimation: A review of constitutive models. European Polymer Journal. 2014;50:97-108. DOI: 10.1016/j.eurpolymj.2013.10.006

[36] ANSYS ${ }^{\circledR}$ Academic Research. Help System // Mechanical APDL // Material Reference // 5. Material Curve Fitting // 5.1. Hyperelastic Material Curve Fitting. Canonsburg (PA), USA; 2013.

[37] Bergstrom J. MCalibration - Fast and powerful material model calibration tool; 2015. http://polymerfem.com/content.php?9-MCalibration Forum: PolymerFEM - Constitutive Models.

[38] Skacel P. Hyperfit - A fitting utility for parameter identification of hyperelastic constitutive models; 2015. http://hyperfit.wz.cz Website: Fitting of Hyperelastic Models.

[39] Wolberg J. Data Analysis Using the Method of Least Squares: Extracting the Most Information from Experiments. Berlin: Springer-Verlag; 2006.

[40] ANSYS, Inc . Calibrating and Validating a Hyperelastic Constitutive Model. In: ANSYS Mechanical APDL Technology Demonstration Guide. Release 15.0 ed. Canonsburg, USA: SAS IP, Inc.; 2013. p. 143-153.

[41] Mars WV, Fatemi A. Observations of the Constitutive Response and Characterization of Filled Natural Rubber Under Monotonic and Cyclic Multiaxial Stress States. J Eng Mat \& Tech. 2004;126(1):19-28. DOI: 10.1115/1.1631432

[42] Gough J, Gregory IH, Muhr AH. Determination of Constitutive Equations for Vulcanized Rubber. In: Boast D, Coveney VA, editors. Finite Element Analysis of Elastomers. London: Professional Engineering Publishing; 1999. p. 5-26.

[43] Dassault Systèmes SolidWorks Corp . SolidWorks 2012 Material Library; 2011. SolidWorks $(R)$ Education Edition, Academic Year 2012-2013. Interactive database of material properties for
SolidWorks Simulation.

[44] Lindley PB, Fuller KNG, Muhr AH. Engineering Design with Natural Rubber. Tun Abdul Razak Laboratory, Brickendonbury, Hertford, UK: The Malaysian Rubber Producers' Research Association; 1992. 5th edition.

[45] Gent AN. Elasticity. In: Gent AN, editor. Engineering with Rubber: How to Design Rubber Components. 3rd ed. Munich: Carl Hanser Verlag; 2012. p. 37-88.

[46] Yeoh OH, Pinter GA, Banks HT. Compression of Bonded Rubber Blocks. Rubber Chem \& Technol. 2002;75(3):549-562. DOI: $10.5254 / 1.3547682$

[47] Sussman T, Bathe $\mathrm{K}$-J. A finite element formulation for nonlinear incompressible elastic and inelastic analysis. Int J Comp \& Struct. 1987;26(1-2):357-409. DOI: $10.1016 / 0045-7949(87) 90265-3$

[48] Young WC, Budynas RG. Roark's Formulas for Stress and Strain. 7th ed. New York, USA: McGraw-Hill Professional; 2001.

[49] Gorash Y, Chen H. A parametric study on creepfatigue strength of welded joints using the linear matching method. Int $\mathbf{J}$ of Fatigue. 2013;55:112-125. DOI: 10.1016/j.ijfatigue.2013.06.011

[50] Gorash Y, Chen H. On creep-fatigue endurance of TIG-dressed weldments using the linear matching method. Eng Failure Analysis. 2013;34:308-323. DOI: $10.1016 /$ j.engfailanal.2013.08.009

[51] Wriggers P. Constitutive Equations for Contact Interfaces. In: Computational Contact Mechanics. Berlin/Heidelberg, Germany: Springer-Verlag; 2006. p. 69-108. DOI: 10.1007/978-3-540-32609-0

[52] Ma W, Qu B, Guan F. Effect of the friction coefficient for contact pressure of packer rubber. Proc IMechE Part C: J Mechanical Engineering Science. 2014;228(16):2881-2887. DOI: $10.1177 / 0954406214525596$

[53] Lindley PB. Compression Characteristics of Laterallyunrestrained Rubber O-rings. J Inst Rubber Ind. 1967;1(Jul/Aug):209-213.

[54] Connolly S. Simulation of the extrusion of rubber O-rings through the gaps in flanges [4th Year Project Thesis]. University of Strathclyde. Dep. of Mechanical \& Aerospace Engineering; 2015. 